\title{
Fast Hyperspectral Soft X-Ray Mapping
}

\author{
Colin M. MacRae ${ }^{1 *}$, N.C. Wilson ${ }^{1}$, A. Torpy ${ }^{1}$ and A.E. Hughes ${ }^{1,2}$ \\ 1. Microbeam Laboratory, CSIRO Mineral Resources, Clayton, Australia. \\ 2. Institute of Frontier Materials, Deakin University, Burwood, Australia. \\ * Corresponding author: colin.macrae@csiro.au
}

X-ray mapping is an important function of both the SEM and EPMA, and in recent decades developments in detectors and computing technology has allowed the collection of hyperspectral maps. The latest hyperspectral detector to become available is the soft x-ray emission spectrometer (SXES) $[1,2]$. This detector collects a limited range of the x-ray spectrum in parallel, but has lowered the minimum energy of detectable $\mathrm{x}$-rays to as low as $45 \mathrm{eV}$, with an energy resolution as low as $99 \mathrm{meV}$ for Mg-L (49 eV) [3]. This not only opens up the opportunity for direct lithium measurement in the microprobe, but also allows for the spectral mapping of L, M, and $\mathrm{N}$ lines of some elements. These low energy lines originate from transitions of electrons from orbitals that are involved in bonding, and thus the peak shape and position of these lines are sensitive to the local chemical environment.

The soft $\mathrm{x}$-rays are dispersed by an aberration corrected varied-line spacing grating onto a $2048 \times 2048$ pixel CCD camera that is sensitive to soft x-rays. However, one drawback in using a CCD with such a large number of pixels is the time taken to readout a spectrum. While on-chip binning can be applied in the direction perpendicular to the energy dispersion to reduce the readout time, typically on currently available SXES systems the fastest pixel speed achievable is $\sim 300$ milliseconds. This is an order of magnitude slower than the typical speed used in EPMA x-ray mapping, meaning that the collection of soft x-rays will greatly extend the time of maps collected in parallel with other signals such as CL, BSE, EDS and WDS. Another drawback of slow readouts, is that the longer acquisition times can induce more sample damage and lead to more sample contamination.

A new generation of CCD cameras built by Princeton Instruments helps solve this speed issue by changing the readout design of the CCD. In a traditional CCD, the CCD pixels are read out of a single port, however new designs effectively split the $2048 \times 2048$ pixel CCD into four $1024 \times 1024$ pixel regions, each with their own readout port. This reduces the CCD readout by reading four CCD pixels simultaneously, as well as having shorter distances on the CCD to move charge. We have fitted a Princeton instruments Sophia-XO-2048B CCD camera to a JEOL SXES detector installed on a fieldemission gun EPMA (JEOL 8530F). The EPMA is also equipped with four WDS detectors, two SDD EDS detectors (Bruker XFLASH 6010) and a grating spectrometer cathodoluminescence system [4]. In the EPMA none of these detectors obscure one another, and so we are able to collect all signals from these detectors in parallel. Employing the quad readout mode of the camera, we have been able to achieve readout speeds down to 30 milliseconds. One downside of faster readout is that less signal is accumulated in the time period, which worsens the signal-to-noise ratio of a measurement. However improved electronics in the Sophia camera has also improved the noise characteristics compared to older models.

Fig. 1 (a) shows a soft x-ray map collected using the Sophia camera of an Al alloy (AA2090) which contains $\sim 2 \mathrm{wt} \% \mathrm{Li}$. The map was collected at $5 \mathrm{kV}$ and $300 \mathrm{nA}$, and is $330 \times 330$ pixels in size, with a pixel dwell of $40 \mathrm{~ms}$, giving a total map time of 1.2 hours. Using previous cameras the map time would 
have been 10 hours or more. By integrating a number of pixels together we average out the read-noise from the individual spectra, and reveal subtle spectral information. The spectrum in Fig 1 (b) taken from a bright area in the map shows not only the Li K edge, but a satellite Li peak [3] at $\sim 46 \mathrm{eV}$ which had not previously been observed in this alloy. A larger map, Fig. 2, with $1500 \mathrm{X} 1500$ pixels, was collected at $5 \mathrm{kV}, 100 \mathrm{nA}$ and $120 \mathrm{~ms}$ dwell time to investigate the Li content of the intermetallic (IM) particles. The copper rich Al IM particles reveal the presence of $\mathrm{Li}$ which is a similar result to previously reported for the AA2099-T8 alloy [5], in addition a satellite peak at $\sim 46 \mathrm{eV}$ is observed [6].

\section{References:}

[1] H Takahashi et al., Microanalysis, Microscopy and Microanalysis 16(S2) (2010), p. 34.

[2] M Terauchi et al., 2012 Journal of Electron Microscopy 61(1) (2012), p. 1.

[3] ET Arakawa and MW Williams, Physical Review B 8(9) (1973), p. 4075.

[4] CM MacRae et al., IOP Conference Series: Materials Science and Engineering 304 (2018), p. 012010.

[5] CM MacRae et al., Microscopy and Microanalysis 24(4) (2018), p. 325.

[6] The authors gratefully acknowledge funding from the ARC LE130100087.
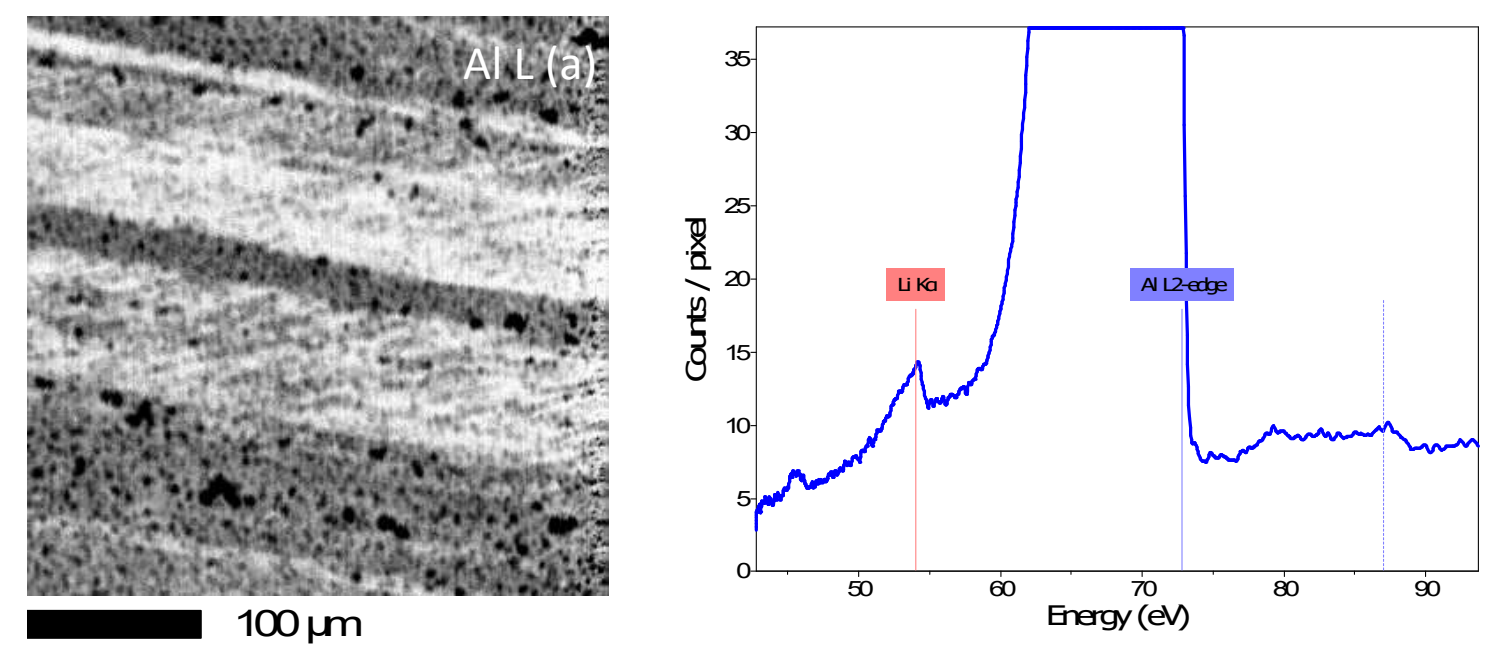

Figure 1. (a) Al L2 edge map of an aluminium alloy containing $\sim 2 \% \mathrm{Li}$, with the map and (b) a spectrum extracted from a bright area showing the Al L, Li K edges and a Li satellite peak at $45.7 \mathrm{eV}$.
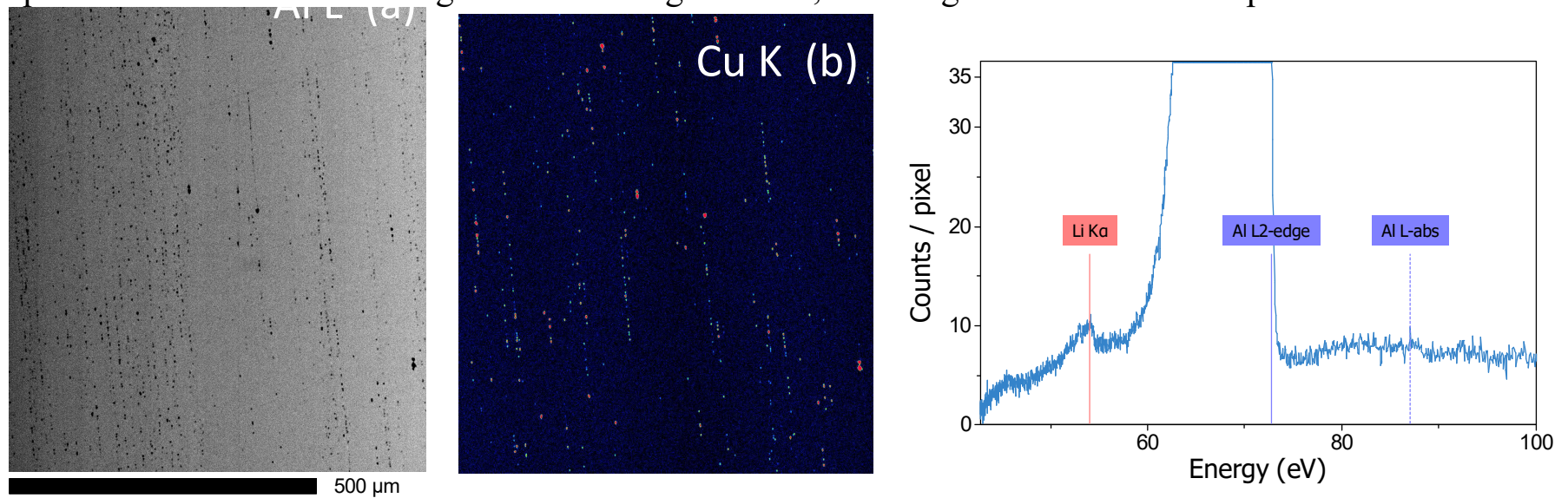

Figure 2. (a) 1500 X 1500 pixel Al L2 edge map of AA2090, (b) Cu K WDS map and (c) SXES spectra from copper rich Al IM particles. The SXES spectral shows the presence of a satellite peak at $\sim 46 \mathrm{eV}$. 